\title{
Design of Half Code Rate Generalized Complex Orthogonal Space Time Block Codes for Multiple Transmit Antennas
}

\author{
Jaipreet $\operatorname{Kaur}^{1}$ and Maninder Lal Singh ${ }^{2}$ \\ ${ }^{1}$ Department of Electronics and Communication Engineering, GNDU RC \\ Sathiala, Amritsar-143205, Punjab, India \\ ${ }^{2}$ Department of Electronics Technology, Guru Nanak Dev University, Amritsar- \\ 143001, Punjab, India \\ ljaipreetkr@yahoo.com, ${ }^{2}$ mlsingh7@gmail.com
}

\begin{abstract}
This paper investigates the maximum likelihood $(M L)$ decoding of complex orthogonal structure of the space time block codes (STBC) having rate 1/2 of multiple input single output (MISO) systems with 2, 3, 4, 5, 6, 7, 8 and 9 transmit antennas. The objective is to explore generator matrices of complex signal constellations that provide high rate as well as full diversity by allowing the simple ML decoding of the symbols.
\end{abstract}

Keywords- Orthogonal space time block codes (OSTBC), maximum likelihood (ML), multiple input single output (MISO, bit error rate (BER), transmit diversity, Alamouti

\section{Introduction}

The most favorable technology in wireless communications is multiple input multiple output (MIMO) systems at the transmitter and/or at the receiver side. The basic idea in MIMO system is space time signal processing by using multiple, spatially spaced antennas at either both link sides or single link side. In space time block codes (STBC), the number of transmitting antennas are equal to the transmitted code symbols per time slot. The efficient space time encoder designs the code symbols by keeping high throughput, diversity gain and coding gain with least decoding complexity $[1,2,3]$. The key design of STBC is dependent on orthogonal designs and was firstly designed by Alamouti [1]. The Alamouti code is for two transmitting antennas and has potential of full diversity and full data rate. The significance of Alamouti is orthogonality of signals transmitted by two transmit antennas which can be generalized to any number of transmit antennas. The extension of the STBC from two transmit antennas to larger number of transmit antenna was proposed by Tarokh, Jafarkhani and Calderbank [3] by relating Hurwitz-Radon theory with orthogonal designs. Hurwitz-Radon theory $[4,5]$ proves that for any number of transmit antennas having rate one can be achieved by real pulse amplitude modulation (PAM) orthogonal designs. Rates cannot be greater than one for complex orthogonal STBC designs as illustrated by [2, 3]. Weifeng and Xiang-Gen [6] demonstrated that rates of more than two transmitting antennas cannot be greater than $3 / 4$ for generalized complex orthogonal designs. Ganesan and Stoica [4] projected the above schemes using maximum signal to noise ratio (SNR). There is no existence of complex valued STBC with full diversity and full data rate for more than two transmit antennas. So, either full diversity or full data rate can be achieved by various proposed code design methods. The STBC for any number of transmit antennas for rate $1 / 2$ can be constructed by generalization of complex quadrature amplitude modulation (QAM), and phase shift keying (PSK) orthogonal designs, which is investigated in present work. With the

Received (February 16, 2018), Review Result (June 28, 2018), Accepted (June 29, 2018) 
application of generalized orthogonal designs, the results of Radon have been extended to both non square and complex orthogonal designs for 3, 4, 5, 6, 7, 8 and 9 transmitting antennas in this paper. This can be extended to larger number of transmit antennas as well.

\section{Orthogonal Space Time Block Coding}

The primary category of linear STBC is orthogonal space time block codes (OSTBC) which leads to simple maximum likelihood (ML) decoding of received symbols as well as provides high diversity gain and coding gain. STBC introduces redundancy in time with the channel coding and redundancies in space with the addition of multiple transmit antennas. The multiple input single output (MISO) system has been considered with $M(=1,2, \ldots, m)$ transmitting antennas and a single receive antenna in this paper. The channel coefficient gains between $i$ th transmitter , $\forall 1 \leq i \leq m$ and receiver is represented by $\lambda(i)$. For the block code of length $T$ and time slot $t(=1,2, \ldots, T)$, the signal transmitted by $i$ th transmitter is represented by $g_{t}^{i}, \forall 1 \leq i \leq m$. The transmit code word $G_{M}$ for $M$ transmit antennas can be given by

$$
G_{M}=\left[\begin{array}{cccc}
g_{1}^{1} & g_{1}^{2} & \cdots & g_{1}^{m} \\
g_{2}^{1} & g_{2}^{2} & \cdots & g_{2}^{m} \\
\vdots & \vdots & \ddots & \vdots \\
g_{T}^{1} & g_{T}^{2} & \cdots & g_{T}^{m}
\end{array}\right]
$$

The receiver receives $r^{t}$ at $t$ th instant for $i$ th transmitter is given by

$$
r^{t}=\sum_{i=1}^{n} \lambda(i) g_{t}^{i}+\mathcal{N}_{t}^{i}
$$

where $\mathcal{N}_{t}^{i}$ is additive white Gaussian noise (AWGN) at $t$ th instant of $i$ th transmitter

The ML detection has been used in this paper as it has the best system performance, but at the same time computational complexity increases exponentially with the symbol constellation size and the number of transmit antennas. The selected code $\widetilde{g}$ during detection is given by

$$
\widetilde{g}=\sum_{t=1}^{T}\left|r^{t}-\sum_{i=1}^{m} \lambda(i) g_{t}^{i}\right|^{2}
$$

The foremost property of OSTBC is the involuntary matrix, obtained by premultiplying the code matrix and the channel matrix with its Hermitian transpose.

A real code orthogonal design matrix $G_{M}(M \times M)$, having real entries $g_{1},-g_{1}, \ldots, g_{M},-g_{M}$ and the channel transfer matrix $\Omega_{M}(M \times M)$, having channel coefficients $\lambda_{1}, \ldots, \lambda_{M}$, satisfy equations (3) and (4) to prove their orthogonality.

$$
\begin{aligned}
& G_{M}{ }^{T} \cdot G_{M}=\left(\left|g_{1}\right|^{2}+\cdots+\left|g_{M}\right|^{2}\right) I_{M} \\
& \Omega_{M} \cdot \Omega_{M}{ }^{T}=\left(\left|\lambda_{1}\right|^{2}+\cdots+\left|\lambda_{M}\right|^{2}\right) I_{M}
\end{aligned}
$$

where $I_{M}$ is the $M \times M$ identity matrix.

A real orthogonal design exists only if $M=2^{x}$, where $x$ is a natural number. A complex code orthogonal design has code rate $K / T$, where $K$ is number of transmitted symbols and $T$ is number of time slots taken by code to transmit/receive symbols. The $M \times T$ complex orthogonal matrix $G_{c M}$ has complex entries $g_{1},-g_{1}, \ldots, g_{K},-g_{K}$ with their conjugates $g_{1}^{*},-g_{1}^{*}, \ldots, g_{K}^{*},-g_{K}^{*}$ and the channel transfer matrix $\Omega_{c M}$ has complex entries $\lambda_{1}, \ldots, \lambda_{K}$ with their conjugates $\lambda_{1}^{*}, \ldots, \lambda_{K}^{*}$ satisfy equations (5) and (6).

$$
G_{c M}{ }^{H} \cdot G_{c M}=\left(\left|g_{1}\right|^{2}+\cdots+\left|g_{K}\right|^{2}\right) I_{M}
$$




$$
\Omega_{c M} \cdot \Omega_{c M}{ }^{H}=\left(\left|\lambda_{1}\right|^{2}+\cdots+\left|\lambda_{K}\right|^{2}\right) I_{M}
$$

The second property of OSTBC is product of orthogonal code design and unitary matrix generates another orthogonal code design. For any orthogonal code design given by $G_{M}=\sum_{k=1}^{K} g_{k} A_{k}$ and unitary matrix $U\left(U^{T} U=I\right)$, the multiplication of them, $U G_{M}=$ $\sum_{k=1}^{K} g_{k} U A_{k}$ is also an orthogonal design.

\section{Alamouti Code}

The Alamouti STBC technique employs two transmitting antennas and one receiving antenna with maximum diversity gain of 2 . This code has full rate, because at every twotime instant, it transmits two symbols. The design of code is given by the generator matrix $G_{c 2}$.

$$
G_{c 2}=\left(\begin{array}{cc}
g_{1} & g_{2} \\
-g_{2}^{*} & g_{1}^{*}
\end{array}\right)
$$

At first time instant, $g_{1}$ and $g_{2}$ symbols are sent by transmitter 1 and transmitter 2 respectively and received vector is given by

$$
r_{1}=\left[\begin{array}{ll}
\lambda_{1} & \lambda_{2}
\end{array}\right]\left[\begin{array}{l}
g_{1} \\
g_{2}
\end{array}\right]+n(1)
$$

At second time instant, $-g_{2}^{*}$ and $g_{1}^{*}$ symbols are sent by transmitter 1 and transmitter 2 respectively and received vector is given by

$$
r_{2}=\left[\begin{array}{ll}
\lambda_{1} & \lambda_{2}
\end{array}\right]\left[\begin{array}{c}
-g_{2}^{*} \\
g_{1}^{*}
\end{array}\right]+n(2)
$$

By combining equation (7) and (8) into a single matrix equation (9)

$$
\begin{array}{r}
{\left[\begin{array}{l}
r_{1} \\
r_{2}^{*}
\end{array}\right]=\left[\begin{array}{cc}
\lambda_{1} & \lambda_{2} \\
\lambda_{2}^{*} & -\lambda_{1}^{*}
\end{array}\right]\left[\begin{array}{l}
g_{1} \\
g_{2}
\end{array}\right]+\left[\begin{array}{c}
n(1) \\
n^{*}(2)
\end{array}\right]} \\
\left(r_{1}, r_{2}^{*}\right)=\left(g_{1}, g_{2}\right) \Omega_{c 2}+\mathcal{N}
\end{array}
$$

where $\mathcal{N}$ is AWGN noise and $\Omega_{c 2}=\left(\begin{array}{cc}\lambda_{1} & \lambda_{2}^{*} \\ \lambda_{2} & -\lambda_{1}^{*}\end{array}\right)$

$$
\begin{aligned}
& G_{c 2}{ }^{H} \cdot G_{c 2}=\left(\left|g_{1}\right|^{2}+\left|g_{2}\right|^{2}\right) I_{2} \\
& \Omega_{c 2} \cdot \Omega_{c 2}{ }^{H}=\left(\left|\lambda_{1}\right|^{2}+\left|\lambda_{2}\right|^{2}\right) I_{2}
\end{aligned}
$$

Multiplying both sides of equation (10) by $\Omega^{H}$ gives

$$
\left(\widetilde{g_{1}}, \widetilde{g_{2}}\right)=\left(r_{1}, r_{2}^{*}\right) \Omega^{H}=\left(\left|\lambda_{1}\right|^{2}+\left|\lambda_{2}\right|^{2}\right)\left(g_{1}, g_{2}\right)+\mathcal{N}
$$

The orthogonality of Alamouti code is proved in equations (11) and (12), resulting in simple ML decoding with full diversity, as shown in equation (13).

\section{The Hurwitz-Radon Theory}

Hurwitz-Radon Theory is used to design generator matrices for more than two transmit antennas with alike properties as of Alamouti code. This theorem is applicable on square matrices only. The realization of non-square matrices is carried out with the help of generalization of orthogonal designs. The real PAM orthogonal space time codes have been constructed for any number of transmit antennas having rate 1 , whereas for the complex QAM, PSK orthogonal space time codes, only rate 1/2 has been constructed for any number of transmit antennas from the real Hurwitz-Radon families. 
The set of $F$ Hurwitz-Radon family of matrices is given by $\left\{A_{2}, A_{3}, \cdots, A_{M}\right\}$, is an $M \times$ $M$ real matrix, satisfying equation (14).

$$
\begin{array}{cc}
A_{l}^{T} \cdot A_{l}=I_{M}, & l=1,2, \cdots, F \\
A_{l}^{T}=-A_{l}, & l=1,2, \cdots, F \\
A_{l} \cdot A_{l},=-A_{l^{\prime}} A_{l}, & 1 \leq l<l^{\prime} \leq F
\end{array}
$$

The maximum size of $M \times M$ Hurwitz-Radon family is determined by:

$$
M=2^{a} b
$$

where $M$ is positive integer, $a=4 c+d$, having $c \geq 0,0 \leq d \leq 4$ and $b$ is an odd number.

The number of $M \times M$ or $\left(2^{a} \times 2^{a}\right)$ Hurwitz-Radon family matrices $F$ is less than $\rho(M)=8 c+2^{d} \leq M$. So the maximum possible size of Hurwitz-Radon family $F$ is $\rho(M)-1$. The various possible values of function $\rho(M)$ is tabulated in Table 1 .

\section{Table 1. Values of Function $\rho(M)$}

\begin{tabular}{ccc}
\hline $\boldsymbol{a}$ & $\boldsymbol{M}=\mathbf{2}^{\boldsymbol{a}}$ & $\boldsymbol{\rho}(\boldsymbol{M})$ \\
\hline 1 & 2 & 2 \\
\hline 2 & 4 & 4 \\
\hline 3 & 8 & 8 \\
\hline 4 & 16 & 9 \\
\hline 5 & 32 & 10 \\
\hline 6 & 64 & 12 \\
\hline 7 & 128 & 16 \\
\hline 8 & 256 & 17 \\
\hline
\end{tabular}

The orthogonal codes are designed for $M=T$ transmit antennas have $\rho(T)$ real symbols. The rate of code $R$ is $\rho(T) / T$.

\section{$2 \times 2$ OSTBC design (Alamouti)}

For $a=1, \rho(M)=2$, the Hurwitz-Radon family size, $T$ is $\rho(2)-1=1$ and Hurwitz-Radon family matrices is given by $R=\left(\begin{array}{cc}0 & 1 \\ -1 & 0\end{array}\right)$. The $2 \times 2$ OSTBC design is given by

$$
G_{2}=g_{1} I_{2}+g_{2} R=\left(\begin{array}{cc}
g_{1} & g_{2} \\
-g_{2} & g_{1}
\end{array}\right)
$$

\section{$4 \times 4$ OSTBC Design}

For $a=2, \rho(M)=4$, the Hurwitz-Radon family size, $T$ is $\rho(4)-1=3$ and HurwitzRadon family matrices are $\left\{A_{2}=Q \otimes R, A_{3}=R \otimes I_{2}, A_{4}=P \otimes R\right\}$.

where, $P=\left(\begin{array}{ll}0 & 1 \\ 1 & 0\end{array}\right), Q=\left(\begin{array}{cc}1 & 0 \\ 0 & -1\end{array}\right)$

$$
\begin{gathered}
A_{2}=\left(\begin{array}{cccc}
0 & 1 & 0 & 0 \\
-1 & 0 & 0 & 0 \\
0 & 0 & 0 & -1 \\
0 & 0 & 1 & 0
\end{array}\right), \quad A_{3}=\left(\begin{array}{ccccc}
0 & 0 & 1 & 0 \\
0 & 0 & 0 & 1 \\
-1 & 0 & 0 & 0 \\
0 & -1 & 0 & 0
\end{array}\right) \\
A_{4}=\left(\begin{array}{cccc}
0 & 0 & 0 & 1 \\
0 & 0 & -1 & 0 \\
0 & 1 & 0 & 0 \\
-1 & 0 & 0 & 0
\end{array}\right)
\end{gathered}
$$


A $4 \times 4$ OSTBC design is given by:

$$
G_{4}=g_{1} I_{4}+g_{2} A_{2}+g_{3} A_{3}+g_{4} A_{4}=\left(\begin{array}{cccc}
g_{1} & g_{2} & g_{3} & g_{4} \\
-g_{2} & g_{1} & -g_{4} & g_{3} \\
-g_{3} & g_{4} & g_{1} & -g_{2} \\
-g_{4} & -g_{3} & g_{2} & g_{1}
\end{array}\right)
$$

\section{$8 \times 8$ OSTBC Design}

For $a=3, \rho(M)=8$, the Hurwitz-Radon family size, $T$ is $\rho(8)-1=7$ and Hurwitz-Radon family matrices are given by $\left\{A_{2}=Q \otimes Q \otimes R, A_{3}=I_{2} \otimes R \otimes I_{2}, A_{4}=\right.$ $\left.I_{2} \otimes P \otimes R, A_{5}=R \otimes Q \otimes I_{2}, A_{6}=P \otimes Q \otimes R, A_{7}=R \otimes P \otimes Q, A_{8}=R \otimes P \otimes P\right\}$

$$
\begin{aligned}
& A_{2}=\left(\begin{array}{cccccccc}
0 & 1 & 0 & 0 & 0 & 0 & 0 & 0 \\
-1 & 0 & 0 & 0 & 0 & 0 & 0 & 0 \\
0 & 0 & 0 & -1 & 0 & 0 & 0 & 0 \\
0 & 0 & 1 & 0 & 0 & 0 & 0 & 0 \\
0 & 0 & 0 & 0 & 0 & -1 & 0 & 0 \\
0 & 0 & 0 & 0 & 1 & 0 & 0 & 0 \\
0 & 0 & 0 & 0 & 0 & 0 & 0 & 1 \\
0 & 0 & 0 & 0 & 0 & 0 & -; 1 & 0
\end{array}\right) A_{3} \\
& =\left(\begin{array}{cccccccc}
0 & 0 & 1 & 0 & 0 & 0 & 0 & 0 \\
0 & 0 & 0 & 1 & 0 & 0 & 0 & 0 \\
-1 & 0 & 0 & 0 & 0 & 0 & 0 & 0 \\
0 & -1 & 0 & 0 & 0 & 0 & 0 & 0 \\
0 & 0 & 0 & 0 & 0 & 0 & 1 & 0 \\
0 & 0 & 0 & 0 & 0 & 0 & 0 & 1 \\
0 & 0 & 0 & 0 & -1 & 0 & 0 & 0 \\
0 & 0 & 0 & 0 & 0 & -1 & 0 & 0
\end{array}\right) \\
& A_{4}=\left(\begin{array}{cccccccc}
0 & 0 & 0 & 1 & 0 & 0 & 0 & 0 \\
0 & 0 & -1 & 0 & 0 & 0 & 0 & 0 \\
0 & 1 & 0 & 0 & 0 & 0 & 0 & 0 \\
-1 & 0 & 0 & 0 & 0 & 0 & 0 & 0 \\
0 & 0 & 0 & 0 & 0 & 0 & 0 & 1 \\
0 & 0 & 0 & 0 & 0 & 0 & -1 & 0 \\
0 & 0 & 0 & 0 & 0 & 1 & 0 & 0 \\
0 & 0 & 0 & 0 & -1 & 0 & 0 & 0
\end{array}\right) \quad A_{5} \\
& =\left(\begin{array}{cccccccc}
0 & 0 & 0 & 0 & 1 & 0 & 0 & 0 \\
0 & 0 & 0 & 0 & 0 & 1 & 0 & 0 \\
0 & 0 & 0 & 0 & 0 & 0 & -1 & 0 \\
0 & 0 & 0 & 0 & 0 & 0 & 0 & -1 \\
-1 & 0 & 0 & 0 & 0 & 0 & 0 & 0 \\
0 & -1 & 0 & 0 & 0 & 0 & 0 & 0 \\
0 & 0 & 1 & 0 & 0 & 0 & 0 & 0 \\
0 & 0 & 0 & 1 & 0 & 0 & 0 & 0
\end{array}\right)
\end{aligned}
$$




$$
\begin{aligned}
& A_{6}=\left(\begin{array}{cccccccc}
0 & 0 & 0 & 0 & 0 & 1 & 0 & 0 \\
0 & 0 & 0 & 0 & -1 & 0 & 0 & 0 \\
0 & 0 & 0 & 0 & 0 & 0 & 0 & -1 \\
0 & 0 & 0 & 0 & 0 & 0 & 1 & 0 \\
0 & 1 & 0 & 0 & 0 & 0 & 0 & 0 \\
-1 & 0 & 0 & 0 & 0 & 0 & 0 & 0 \\
0 & 0 & 0 & -1 & 0 & 0 & 0 & 0 \\
0 & 0 & 1 & 0 & 0 & 0 & 0 & 0
\end{array}\right) \quad A_{7} \\
& =\left(\begin{array}{cccccccc}
0 & 0 & 0 & 0 & 0 & 0 & 1 & 0 \\
0 & 0 & 0 & 0 & 0 & 0 & 0 & -1 \\
0 & 0 & 0 & 0 & 1 & 0 & 0 & 0 \\
0 & 0 & 0 & 0 & 0 & -1 & 0 & 0 \\
0 & 0 & -1 & 0 & 0 & 0 & 0 & 0 \\
0 & 0 & 0 & 1 & 0 & 0 & 0 & 0 \\
-1 & 0 & 0 & 0 & 0 & 0 & 0 & 0 \\
0 & 1 & 0 & 0 & 0 & 0 & 0 & 0
\end{array}\right) \\
& A_{8}=\left(\begin{array}{cccccccc}
0 & 0 & 0 & 0 & 0 & 0 & 0 & 1 \\
0 & 0 & 0 & 0 & 0 & 0 & 1 & 0 \\
0 & 0 & 0 & 0 & 0 & 1 & 0 & 0 \\
0 & 0 & 0 & 0 & 1 & 0 & 0 & 0 \\
0 & 0 & 0 & -1 & 0 & 0 & 0 & 0 \\
0 & 0 & -1 & 0 & 0 & 0 & 0 & 0 \\
0 & -1 & 0 & 0 & 0 & 0 & 0 & 0 \\
-1 & 0 & 0 & 0 & 0 & 0 & 0 & 0
\end{array}\right)
\end{aligned}
$$

A $8 \times 8$ OSTBC design $G_{8}$, and its channel matrix $\Omega_{8}$, which satisfies the orthogonal condition given in equation, is written as

$$
\begin{gathered}
G_{8}=g_{1} I_{8}+g_{2} A_{2}+g_{3} A_{3}+g_{4} A_{4}+g_{5} A_{5}+g_{6} A_{6}+g_{7} A_{7}+g_{8} A_{8} \\
G_{8}=\left(\begin{array}{llllllll}
g_{1} & g_{2} & g_{3} & g_{4} & g_{5} & g_{6} & g_{7} & g_{8} \\
-g_{2} & g_{1} & -g_{4} & g_{3} & -g_{6} & g_{5} & g_{8} & -g_{7} \\
-g_{3} & g_{4} & g_{1} & -g_{2} & g_{7} & g_{8} & -g_{5} & -g_{6} \\
-g_{4} & -g_{3} & g_{2} & g_{1} & g_{8} & -g_{7} & g_{6} & -g_{5} \\
-g_{5} & g_{6} & -g_{7} & -g_{8} & g_{1} & -g_{2} & g_{3} & g_{4} \\
-g_{6} & -g_{5} & -g_{8} & g_{7} & g_{2} & g_{1} & -g_{4} & g_{3} \\
-g_{7} & -g_{8} & g_{5} & -g_{6} & -g_{3} & g_{4} & g_{1} & g_{2} \\
-g_{8} & g_{7} & g_{6} & g_{5} & -g_{4} & -g_{3} & -g_{2} & g_{1}
\end{array}\right) \\
\Omega_{8}=\left(\begin{array}{llllllll}
\lambda_{1} & \lambda_{2} & \lambda_{3} & \lambda_{4} & \lambda_{5} & \lambda_{6} & \lambda_{7} & \lambda_{8} \\
\lambda_{2} & -\lambda_{1} & -\lambda_{4} & \lambda_{3} & -\lambda_{6} & \lambda_{5} & \lambda_{8} & -\lambda_{7} \\
\lambda_{3} & \lambda_{4} & -\lambda_{1} & -\lambda_{2} & \lambda_{7} & \lambda_{8} & -\lambda_{5} & -\lambda_{6} \\
\lambda_{4} & -\lambda_{3} & \lambda_{2} & -\lambda_{1} & \lambda_{8} & -\lambda_{7} & \lambda_{6} & -\lambda_{5} \\
\lambda_{5} & \lambda_{6} & -\lambda_{7} & -\lambda_{8} & -\lambda_{1} & -\lambda_{2} & \lambda_{3} & \lambda_{4} \\
\lambda_{6} & -\lambda_{5} & -\lambda_{8} & \lambda_{7} & \lambda_{2} & -\lambda_{1} & -\lambda_{4} & \lambda_{3} \\
\lambda_{7} & -\lambda_{8} & \lambda_{5} & -\lambda_{6} & -\lambda_{3} & \lambda_{4} & -\lambda_{1} & \lambda_{2} \\
\lambda_{8} & \lambda_{7} & \lambda_{6} & \lambda_{5} & -\lambda_{4} & -\lambda_{3} & -\lambda_{2} & -\lambda_{1}
\end{array}\right) \\
G_{8}{ }^{T} \cdot G_{8}=\left(\sum_{n=1}^{8}\left|g_{n}\right|^{2}\right) I_{8}, \Omega_{8} . \Omega_{8}{ }^{T}=\left(\sum_{n=1}^{8}\left|\lambda_{n}\right|^{2}\right) I_{8}
\end{gathered}
$$

\section{$16 \times 9$ OSTBC Design}

Similarly, the $16 \times 9$ OSTBC design for 9 transmit antennas is given by 


$$
G_{9}=\left(\begin{array}{ccccccccc}
g_{1} & g_{2} & g_{3} & g_{4} & g_{5} & g_{6} & g_{7} & g_{8} & -g_{9} \\
-g_{2} & g_{1} & -g_{4} & g_{3} & -g_{6} & g_{5} & g_{8} & -g_{7} & -g_{10} \\
-g_{3} & g_{4} & g_{1} & -g_{2} & g_{7} & g_{8} & -g_{5} & -g_{6} & -g_{11} \\
-g_{4} & -g_{3} & g_{2} & g_{1} & g_{8} & -g_{7} & g_{6} & -g_{5} & -g_{12} \\
-g_{5} & g_{6} & -g_{7} & -g_{8} & g_{1} & -g_{2} & g_{3} & g_{4} & -g_{13} \\
-g_{6} & -g_{5} & -g_{8} & g_{7} & g_{2} & g_{1} & -g_{4} & g_{3} & -g_{14} \\
-g_{7} & -g_{8} & g_{5} & -g_{6} & -g_{3} & g_{4} & g_{1} & g_{2} & -g_{15} \\
-g_{8} & g_{7} & g_{6} & g_{5} & -g_{4} & -g_{3} & -g_{2} & g_{1} & -g_{16} \\
-g_{9} & g_{10} & g_{11} & g_{12} & g_{13} & g_{14} & g_{15} & g_{16} & g_{1} \\
-g_{10} & g_{9} & g_{12} & -g_{11} & g_{14} & -g_{13} & -g_{16} & g_{15} & g_{2} \\
-g_{11} & g_{12} & -g_{9} & g_{10} & -g_{15} & -g_{16} & g_{13} & g_{14} & g_{3} \\
-g_{12} & g_{11} & -g_{10} & -g_{9} & -g_{16} & g_{15} & -g_{14} & g_{13} & g_{4} \\
-g_{13} & g_{14} & g_{15} & g_{16} & -g_{9} & g_{10} & -g_{11} & -g_{12} & g_{5} \\
-g_{14} & g_{13} & g_{16} & -g_{15} & -g_{10} & -g_{9} & g_{12} & -g_{11} & g_{6} \\
-g_{15} & g_{16} & -g_{13} & g_{14} & g_{11} & -g_{12} & -g_{9} & -g_{10} & g_{7} \\
-g_{16} & g_{15} & -g_{14} & -g_{13} & g_{12} & g_{11} & g_{10} & -g_{9} & g_{8}
\end{array}\right)
$$

Table 2. Comparison of OSTBC of $M=2$ to 9 Transmit Antennas having a Receive Antenna

\begin{tabular}{cccc}
\hline OSTBC & $\begin{array}{c}\text { Number of transmit } \\
\text { antennas }(\boldsymbol{N})\end{array}$ & $\begin{array}{c}\text { Number of transmit } \\
\text { symbols }(\boldsymbol{l})\end{array}$ & $\begin{array}{c}\text { Number of time } \\
\text { slots }(\boldsymbol{T})\end{array}$ \\
\hline $\boldsymbol{G}_{\boldsymbol{2}}$ & 2 & 2 & 2 \\
\hline $\boldsymbol{G}_{\mathbf{3}}$ & 3 & 4 & 8 \\
\hline $\boldsymbol{G}_{\mathbf{4}}$ & 4 & 4 & 8 \\
\hline $\boldsymbol{G}_{\mathbf{5}}$ & 5 & 8 & 16 \\
\hline $\boldsymbol{G}_{\mathbf{6}}$ & 6 & 8 & 16 \\
\hline $\boldsymbol{G}_{\mathbf{7}}$ & 7 & 8 & 16 \\
\hline $\boldsymbol{G}_{\mathbf{8}}$ & 8 & 8 & 16 \\
\hline $\boldsymbol{G}_{\mathbf{9}}$ & 9 & 16 & 32 \\
\hline
\end{tabular}

The sub matrix of $G_{8}$ is kept in $G_{9}$ as such and the symbols $g_{9}$ to $g_{16}$ are added in the ninth column of $G_{9}$, then the entries in $G_{9}$ are arranged from ninth row to end such that all nine columns are orthogonal to each other, satisfying $G_{9}{ }^{T} \cdot G_{9}=\left(\sum_{n=1}^{16}\left|g_{n}\right|^{2}\right) I_{9}$. The generalized complex orthogonal designs for any number of transmit antennas can be designed by same procedure. The properties of OSTBC are satisfied by the above constructed generator matrices, $G_{2}, G_{4}, G_{8}$ and $G_{9}$. The OSBTC generator matrix of a smaller number of transmit antennas can be obtained by deleting additional columns from the greater OSTBC generator matrix of same rate. By deleting single column from an orthogonal design produces another orthogonal design having one less transmit antenna with respect to the preceding one. The parameters of OSTBC are summarized in Table 2.

\section{Generalized Complex Orthogonal Design}

The design of complex orthogonal code $G_{c M}$ is formed by combination of a corresponding real orthogonal code design of generator matrix $G_{M}$ with its conjugate.

$$
G_{c M}=\left(\begin{array}{c}
G_{M} \\
G_{M}^{*}
\end{array}\right)=\left(\begin{array}{l}
\sum_{k=1}^{K} g_{k} A_{k} \\
\sum_{k=1}^{K} g_{k}^{*} A_{k}^{*}
\end{array}\right), G_{c M}^{H} \cdot G_{c M}=2 \sum_{k=1}^{K}\left|g_{k}\right|^{2} I_{M}
$$

For three transmit antennas the complex OSTBC matrix $G_{c 3}$, having rate $1 / 2$, has four complex symbols are transmitted during eight time slots. Similarly, for four transmit 
antennas the complex matrix $G_{c 4}$, four symbols are taken at a time and transmitted during eight time slots, with transmission rate of $1 / 2$.

$$
G_{c 3}=\left[\begin{array}{ccc}
g_{1} & g_{2} & g_{3} \\
-g_{2} & g_{1} & -g_{4} \\
-g_{3} & g_{4} & g_{1} \\
-g_{4} & -g_{3} & g_{2} \\
g_{1}^{*} & g_{2}^{*} & g_{3}^{*} \\
-g_{2}^{*} & g_{1}^{*} & -g_{4}^{*} \\
-g_{3}^{*} & g_{4}^{*} & g_{1}^{*} \\
-g_{4}^{*} & -g_{3}^{*} & g_{2}^{*}
\end{array}\right] \quad G_{c 4}=\left[\begin{array}{cccc}
g_{1} & g_{2} & g_{3} & g_{4} \\
-g_{2} & g_{1} & -g_{4} & g_{3} \\
-g_{3} & g_{4} & g_{1} & -g_{2} \\
-g_{4} & -g_{3} & g_{2} & g_{1} \\
g_{1}^{*} & g_{2}^{*} & g_{3}^{*} & g_{4}^{*} \\
-g_{2}^{*} & g_{1}^{*} & -g_{4}^{*} & g_{3}^{*} \\
-g_{3}^{*} & g_{4}^{*} & g_{1}^{*} & -g_{2}^{*} \\
-g_{4}^{*} & -g_{3}^{*} & g_{2}^{*} & g_{1}^{*}
\end{array}\right]
$$

Similarly, the following complex orthogonal designs for $N=5,6,7,8$ and 9 transmit antennas given by $G_{c 5}, G_{c 6}, G_{c 7}, G_{c 8}$ and $G_{c 9}$ respectively. These matrices are obtained by row-wise concatenating the real orthogonal matrix with the complex conjugate of entire real orthogonal matrix.

$$
G_{c 5}=\left[\begin{array}{ccccc}
g_{1} & g_{2} & g_{3} & g_{4} & g_{5} \\
-g_{2} & g_{1} & g_{4} & -g_{3} & g_{6} \\
-g_{3} & -g_{4} & g_{1} & g_{2} & g_{7} \\
-g_{4} & g_{3} & -g_{2} & g_{1} & g_{8} \\
-g_{5} & -g_{6} & -g_{7} & -g_{8} & g_{1} \\
-g_{6} & g_{5} & -g_{8} & g_{7} & -g_{2} \\
-g_{7} & g_{8} & g_{5} & -g_{6} & -g_{3} \\
-g_{8} & -g_{7} & g_{6} & g_{5} & -g_{4} \\
g_{1}^{*} & g_{2}^{*} & g_{3}^{*} & g_{4}^{*} & g_{5}^{*} \\
-g_{2}^{*} & g_{1}^{*} & g_{4}^{*} & -g_{3}^{*} & g_{6}^{*} \\
-g_{3}^{*} & -g_{4}^{*} & g_{1}^{*} & g_{2}^{*} & g_{7}^{*} \\
-g_{4}^{*} & g_{3}^{*} & -g_{2}^{*} & g_{1}^{*} & g_{8}^{*} \\
-g_{5}^{*} & -g_{6}^{*} & -g_{7}^{*} & -g_{8}^{*} & g_{1}^{*} \\
-g_{6}^{*} & g_{5}^{*} & -g_{3}^{*} & g_{7}^{*} & -g_{2}^{*} \\
-g_{7}^{*} & g_{8}^{*} & g_{5}^{*} & -g_{6}^{*} & -g_{3}^{*} \\
-g_{8}^{*} & -g_{7}^{*} & g_{6}^{*} & g_{5}^{*} & -g_{4}^{*}
\end{array}\right] \quad G_{c 6}=\left[\begin{array}{cccccc}
g_{1} & g_{2} & g_{3} & g_{4} & g_{5} & g_{6} \\
-g_{2} & g_{1} & g_{4} & -g_{3} & g_{6} & -g_{5} \\
-g_{3} & -g_{4} & g_{1} & g_{29} & g_{7} & -g_{8} \\
-g_{4} & g_{3} & -g_{2} & g_{1} & g_{8} & -g_{7} \\
-g_{5} & -g_{6} & -g_{7} & -g_{8} & g_{1} & g_{2} \\
-g_{6} & g_{5} & -g_{8} & g_{7} & -g_{2} & g_{1} \\
-g_{7} & g_{8} & g_{5} & -g_{6} & -g_{3} & g_{4} \\
-g_{8} & -g_{7} & g_{6} & g_{5} & -g_{4} & -g_{3} \\
g_{1}^{*} & g_{2}^{*} & g_{3}^{*} & g_{4}^{*} & g_{5}^{*} & g_{6}^{*} \\
-g_{2}^{*} & g_{1}^{*} & g_{4}^{*} & -g_{3}^{*} & g_{6}^{*} & -g_{5}^{*} \\
-g_{3}^{*} & -g_{4}^{*} & g_{1}^{*} & g_{2}^{*} & g_{7}^{*} & -g_{8}^{*} \\
-g_{4}^{*} & g_{3}^{*} & -g_{2}^{*} & g_{1}^{*} & g_{8}^{*} & -g_{7}^{*} \\
-g_{5}^{*} & -g_{6}^{*} & -g_{7}^{*} & -g_{8}^{*} & g_{1}^{*} & g_{2}^{*} \\
-g_{6}^{*} & g_{5}^{*} & -g_{8}^{*} & g_{7}^{*} & -g_{2}^{*} & g_{1}^{*} \\
-g_{7}^{*} & g_{8}^{*} & g_{5}^{*} & -g_{6}^{*} & -g_{3}^{*} & g_{4}^{*} \\
-g_{8}^{*} & -g_{7}^{*} & g_{6}^{*} & g_{5}^{*} & -g_{4}^{*} & -g_{3}^{*}
\end{array}\right]
$$

$$
G_{c 7}=\left[\begin{array}{ccccccc}
g_{1} & g_{2} & g_{3} & g_{4} & g_{5} & g_{6} & g_{7} \\
-g_{2} & g_{1} & g_{4} & -g_{3} & g_{6} & -g_{5} & -g_{8} \\
-g_{3} & -g_{4} & g_{1} & g_{2} & g_{7} & g_{8} & -g_{5} \\
-g_{4} & g_{3} & -g_{2} & g_{1} & g_{8} & -g_{7} & g_{6} \\
-g_{5} & -g_{6} & -g_{7} & -g_{8} & g_{1} & g_{2} & g_{3} \\
-g_{6} & g_{5} & -g_{8} & g_{7} & -g_{2} & g_{1} & -g_{4} \\
-g_{7} & g_{8} & g_{5} & -g_{6} & -g_{3} & g_{4} & g_{1} \\
-g_{8} & -g_{7} & g_{6} & g_{5} & -g_{4} & g_{3} & g_{2} \\
g_{1}^{*} & g_{2}^{*} & g_{3}^{*} & g_{4}^{*} & g_{5}^{*} & g_{6}^{*} & g_{7}^{*} \\
-g_{2}^{*} & g_{1}^{*} & g_{4}^{*} & -g_{3}^{*} & g_{6}^{*} & -g_{5}^{*} & -g_{8}^{*} \\
-g_{3}^{*} & -g_{4}^{*} & g_{1}^{*} & g_{2}^{*} & g_{7}^{*} & g_{8}^{*} & -g_{5}^{*} \\
-g_{4}^{*} & g_{3}^{*} & -g_{2}^{*} & g_{1}^{*} & g_{8}^{*} & -g_{7}^{*} & g_{6}^{*} \\
-g_{5}^{*} & -g_{6}^{*} & -g_{7}^{*} & -g_{8}^{*} & g_{1}^{*} & g_{2}^{*} & g_{3}^{*} \\
-g_{6}^{*} & g_{5}^{*} & -g_{8}^{*} & g_{7}^{*} & -g_{2}^{*} & g_{1}^{*} & -g_{4}^{*} \\
-g_{7}^{*} & g_{8}^{*} & g_{5}^{*} & -g_{6}^{*} & -g_{3}^{*} & g_{4}^{*} & g_{1}^{*} \\
-g_{8}^{*} & -g_{7}^{*} & g_{6}^{*} & g_{5}^{*} & -g_{4}^{*} & g_{3}^{*} & g_{2}^{*}
\end{array}\right]
$$




\section{Simulation Results}

The MISO system is considered with 2,3,4,5,6,7,8 and 9 transmit antennas over Rayleigh fading channels. Figure 1 and Table 3 shows the simulation results for BER performance versus SNR of the complex orthogonal designs for $M=2,3,4,5,6,7,8$ and 9 transmit antennas as given by $G_{c 2}, G_{c 3}, G_{c 4}, G_{c 5}, G_{c 6}, G_{c 7}, G_{c 8}$ and $G_{c 9}$ using one receiver. The system throughput at $1 \mathrm{bit} / \mathrm{sec}$ has been fixed, where BPSK constellation for $G_{c 2}$ and QPSK for other half rate OSTBC like $G_{c 3}, G_{c 4}, G_{c 5}, G_{c 6}, G_{c 7}, G_{c 8}$ and $G_{c 9}$ are considered.

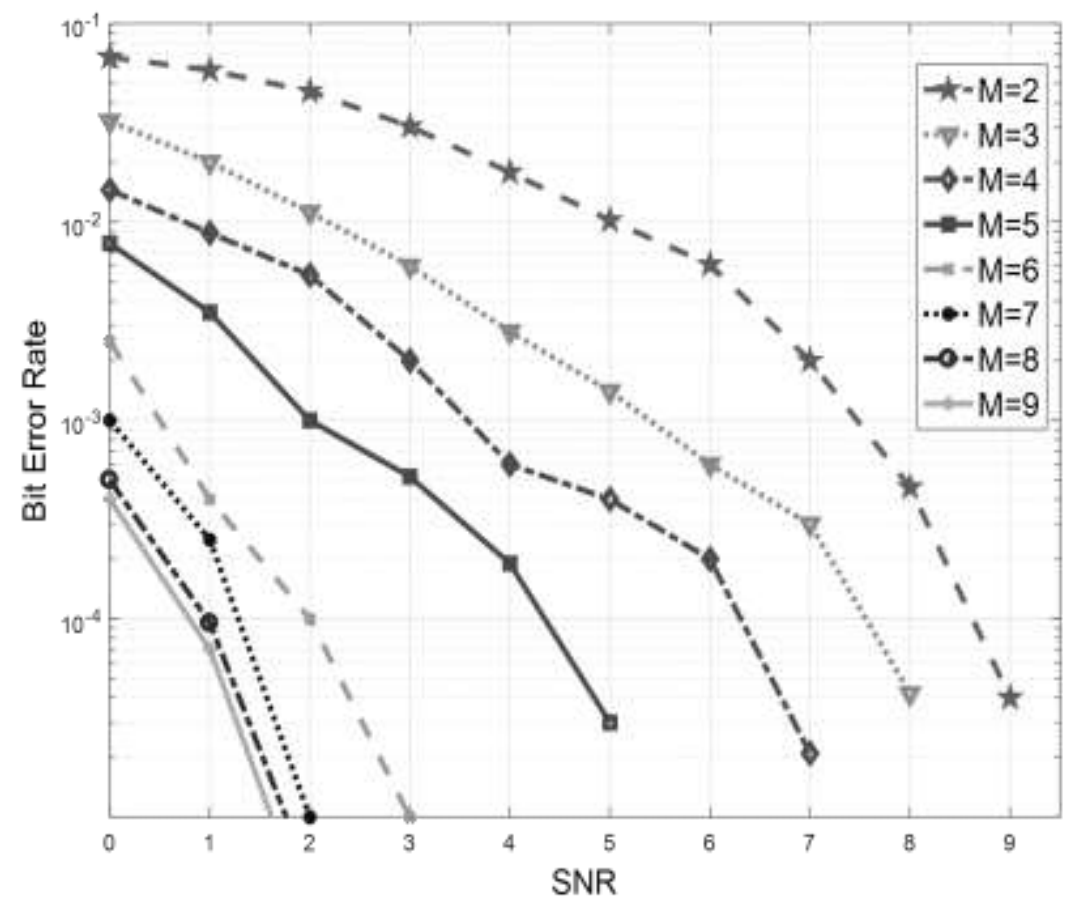

Figure 1. Demonstration of BER versus SNR of the Complex Orthogonal Designs having rate $1 / 2$ for $M=2,3,4,5,6,7,8$ and 9 Transmit Antennas using One Receiver with $1 \mathrm{bit} / \mathrm{sec}$

Table 3. Required SNR (dB) and Gain (dB) of Different OSTBC with Transmission Rate of $1 \mathrm{bit} / \mathrm{sec}$ using One Receiver

\begin{tabular}{|c|c|c|c|c|c|}
\hline \multirow[b]{2}{*}{ Bit/Sec } & \multirow[b]{2}{*}{ Code } & \multirow[b]{2}{*}{ Code Rate } & \multirow[b]{2}{*}{ Modem } & \multicolumn{2}{|c|}{ BER } \\
\hline & & & & $\begin{array}{c}\text { Required SNR }(\mathrm{dB}) \\
\text { at } 10^{-4} \text { Threshold }\end{array}$ & $\begin{array}{l}\text { Successive Gain } \\
\text { Margin }(\mathrm{dB}) \text { at } 10^{-4}\end{array}$ \\
\hline \multirow[t]{8}{*}{1} & $G_{c 2}$ & 1 & BPSK & 8.6 & 0 \\
\hline & $G_{c 3}$ & $1 / 2$ & QPSK & 7.5 & 1.1 \\
\hline & $G_{c 4}$ & $1 / 2$ & QPSK & 6.4 & 0.9 \\
\hline & $G_{c 5}$ & $1 / 2$ & QPSK & 4.4 & 1.0 \\
\hline & $G_{c 6}$ & $1 / 2$ & QPSK & 2 & 2.4 \\
\hline & $G_{c 7}$ & $1 / 2$ & QPSK & 1.2 & 0.8 \\
\hline & $G_{c 8}$ & $1 / 2$ & QPSK & 1 & 0.2 \\
\hline & $G_{c 9}$ & $1 / 2$ & QPSK & 0.9 & 0.1 \\
\hline
\end{tabular}


Similarly, to fix the system throughput at $2 \mathrm{bit} / \mathrm{sec}$, QPSK for $G_{c 2}$ and, 16QAM for other half rate OSTBC like $G_{c 3}, G_{c 4}, G_{c 5}, G_{c 6}, G_{c 7}, G_{c 8}$ and $G_{c 9}$ can be considered. In the same terms for $3 \mathrm{bit} / \mathrm{sec}$, 16QAM for $G_{c 2}$ and 64QAM for remaining can be used.

\section{Conclusion}

The simulation results demonstrate that a significant gain can be achieved by increasing the number of transmit antennas with very little decoding complexity. It has been proved that the generalized real orthogonal design exists for any number of transmit antennas having transmission rate equals to unity and the generalized complex orthogonal design exists for any number of transmit antennas having half of maximum transmission rate. The generalized complex orthogonal design provides full diversity and simple ML decoding. Due to linear processing of ML decoding, the decoding complexity does not increase significantly with the increase of the number of transmit antennas.

\section{References}

[1] S. Alamouti, "A Simple Transmit Diversity Technique for Wireless Communications", IEEE J.Select. Areas Commun., vol. 16, no. 8, (1998), pp. 1451-1458.

[2] V. Tarokh, H. Jafarkhani and A. R. Calderbank, "Space-time codes high data rate wireless communication: performance crirterion and construction", IEEE Trans. on Information Theory, vol. 44, no. 2, (1998) March, pp. 744-765.

[3] V. Tarokh, H. Jafarkhani and A. R. Calderbank, "Space-time block codes from orthogonal designs", IEEE Trans. on Information Theory, vol. 45, no. 5, (1999) July, pp. 1456-1467.

[4] G. Ganesan and P. Stoica, "Space-time Block Codes: A Maximum SNR Approach", IEEE Trans. Inform. Theory, vol. 47, no. 4, (2001), pp. 1650-1656.

[5] J. Seberry, S. Spence and T. A. Wysocki, "A construction technique for generalized complex orthogonal designs and applications to wireless communications", Linear algebra and its applications, (2005), pp. 163-176.

[6] W. Su and X.-G. Xia, "On Space-Time Block Codes from Complex Orthogonal Designs", Wireless Personal Communications, vol. 25, no. 1, (2003), pp. 1-26.

[7] L. Xian and H. Liu, "Rate-One Space-Time Block Codes with Full Diversity", IEEE Trans. Commun, vol. 53 , no. 12 , (2005).

[8] E. A. D. Gespert, "From theory to practice: An overview of MIMO Space Time Coded Wireless Systems", IEEE JSAC, vol. 21, (2003).

[9] W. Su and X.-G. Xia, "Two Generalized Complex Orthogonal Space Time BlockCodes of Rates 7/11 and $3 / 5$ for 5 and 6 Transmit Antennas", IEEE Transactions on Information Theory, vol. 49, no. 1, (2003) January, pp. 313-315.

[10] S. Singh Chauhan and S. Kumar, "Performance Analysis of Multiuser Diversity on OSTBC MIMO Systems with Antenna Selection in the Presence of Feedback Delay CSI", Wireless Personal Communications, vol. 92, no. 2, (2017), pp. 695-710.

[11] G. Qian, P. Wei, Z. Ruan and J. Lu, "A Low-Complexity Modulation Classification Algorithm for MIMO-OSTBC System”, Circuits, Systems, and Signal Processing, vol. 36, no. 6, (2017), pp. 26222634.

[12] S. Kumar Bandari, V. V. Mani and A. Drosopoulos, "Robust Precoded OSTBC for GFDM Systems", Procedia Computer Science, vol. 93, (2016), pp. 176-182.

[13] Z. Veljovic and U. Urosevic, "New Solutions for Cooperative Relaying Implementation of OSTBC with 3/4 Code Rate", Wireless Personal Communications, vol. 92, no. 1, (2017), pp. 51-61. 